\title{
Second degree generalized gauss-Seidel iteration method for solving linear system of equations
}

\author{
Tesfaye Kebede \\ Bahir Dar University, College of Science, Department of Mathematics \\ $\underline{\text { tk_ke@yahoo.com }}$
}

\begin{abstract}
In this paper, a second degree generalized Gauss -Seidel iteration (SDGGS) method for solving linear system of equations whose iterative matrix has real and complex eigenvalues are less than unity in magnitude is presented. Few numerical examples are considered to show the efficiency of the new method compared to first degree Gauss-Seidel (GS), first degree Generalized Gauss-Seidel (GGS) and Second degree Gauss-Seidel (SDGS) methods. It is observed that the spectral radius of the new Second degree Generalized Gauss-Seidel (SDGGS) method is less than the spectral radius of the methods GS, GGS and SDGS. By use of second degree iteration (SD) method, it is possible to accelerate the convergence of any iterative method.
\end{abstract}

Key words: Gauss-Seidel method (GS); Generalized Gauss-Seidel method (GGS); Strictly Diagonally Dominant Matrix.

DOI: http://dx.doi.org/10.4314/ejst.v7i2.5

\section{INTRODUCTION}

Consider a class of linear stationary second degree methods for solving linear system

$$
\mathrm{Ax}=\mathrm{b}
$$

where $\mathrm{A}$ is a given real non singular $n \times n$ matrix and $b$ is a given vector or $\mathrm{nx} 1$ (column) matrix (Saad ,1995). Iterative methods, based on splitting A into

A = D-L-U, compute successive approximations $X^{(n)} X^{(n)}$ to obtain more accurate solutions to a linear system at each iteration step $n$. This process can be written in the form of the general iteration matrix equation as

$$
x^{(n+1)}=G_{1} x^{(n)}+C
$$

In numerical linear algebra the Gauss-Seidel method, also known as the Liebmann method or the method of successive displacement, is an iterative method used to solve a linear system of equations (Kahan, 1958). It is named after the German mathematicians Carl Friedrich Gauss and Philipp Ludwig Von Seidel, and is similar to the Jacobi method. Though it can be applied to any matrix with non-zero elements on the diagonals, convergence is only guaranteed if the matrix is either diagonally dominant, or symmetric and positive definite. The computed Gauss-Seidel iterate successively for each component. It has been proved that, if A is strictly diagonally dominant (SDD) or irreducibly diagonally dominant, then the associated Jacobi and Gauss-Seidel iterations converge for any initial guess $\mathrm{X}^{(0)}(\mathrm{Li}, 2005)$. If $\mathrm{A}$ is symmetric positive definite (SPD) matrix, then the Gauss-Seidel method also converges for any initial guess $\mathrm{X}^{(0)}$ (David, 2007).

The Gauss-Seidel iteration (GS) method for first degree is

$$
x^{(n+1)}=(D-L)^{-1} U x^{(n)}+(D-L)^{-1} b
$$


The Generalized Gauss -Seidel (GGS) iterative method for first degree stated by (David, 2007) is

$$
x^{(n+1)}=\left(D_{m}-L_{m}\right)^{-1} U_{m} x^{(n)}+\left(D_{m}-L_{m}\right)^{-1} b
$$

where, $G_{m}=\left(D_{m}-L_{m}\right)^{-1} U_{m}$ is the iteration matrix of the GGS method.

$$
C=\left(D_{m}-L_{m}\right)^{-1} b \text { is a column vector. }
$$

In the next section, a review of Second degree generalized Gauss-Seidel iterative method (SDGGS) is presented. Following this, the relationship between spectral radius of first degree Gauss-Seidel (GS), first degree generalized Gauss-Seidel (GGS) and Second degree Gauss-Seidel (SDGS) methods and Second degree generalized Gauss-Seidel iteration methods is given. Finally, based on the results on the numerical examples considered, discussion and conclusion made.

\section{SECOND DEGREE GENERALIZED GAUSS-SEIDEL ITERATIVE METHOD}

The linear stationary second degree method is given by (David, 1970) is

$$
x^{(n+1)}=x^{(n)}+a_{1}\left(x^{(n)}-x^{(n-1)}\right)+b_{1}\left(x^{(n+1)}-x^{(n)}\right)
$$

Here, $\mathrm{X}^{(\mathrm{n}+1)}$ appearing in the right hand side as given in (2) is completely consistent for any constant $\mathrm{a}_{1}$ and $\mathrm{b}_{1}$ such that $\mathrm{b}_{1} \neq 0$.

$$
\begin{aligned}
& x^{(n+1)}=x^{(n)}+a_{1}\left(x^{(n)}-x^{(n-1)}\right)+b_{1}\left(G_{1} x^{(n)}+C-x^{(n)}\right) \\
& x^{(n+1)}=x^{(n)}+a_{1} x^{(n)}-a_{1} x^{(n-1)}+b_{1} G_{1} x^{(n)}+b_{1} C-b_{1} x^{(n)} \\
& x^{(n+1)}=\left[\left(1+a_{1}-b_{1}\right) I+b_{1} G_{1}\right] x^{(n)}-a_{1} x^{(n-1)}+b_{1} C \\
& \text { Therefore, } x^{(n+1)}=G x^{(n)}+H x^{(n-1)}+K
\end{aligned}
$$

Where $G=\left(1+a_{1}-b_{1}\right) I+b_{1} G_{1}$

$$
\begin{aligned}
& H=-a_{1} I \\
& K=b_{1} C
\end{aligned}
$$

Theorem1.1:- If matrix A is strictly diagonally dominant, then the associated generalized Gauss-Seidel iteration method converges for any initial approximation, $\mathrm{x}^{(0)}$.

Proof: Since matrix $A$ is strictly diagonally dominant, we have the iteration matrix $\quad G_{m}=\left(D_{m}-L_{m}\right)^{-1} U_{m}$. Taking the norm at infinity of both sides we have

$$
\left\|G_{m}\right\|_{\infty}=\left\|\left(D_{m}-L_{m}\right)^{-1} U_{m}\right\|_{\infty}<\left\|\left(D_{m}-L_{m}\right)^{-1}\right\|_{\infty}\left\|U_{m}\right\|_{\infty}=\left\|\left(D_{m}-L_{m}\right)\right\|_{\infty}^{-1} \cdot\left\|U_{m}\right\|_{\infty}=\frac{\left\|U_{m}\right\|_{\infty}}{\left\|D_{m}-L_{m}\right\|_{\infty}}<1 .
$$

That is $\left\|G_{m}\right\|_{\infty}<1$.

Thus, the generalized Gauss-Seidel iterative method converges for any initial approximation $x^{(0)}$.

The second degree generalized Gauss - Seidel (GGS) method is defined as 


$$
x^{(n+1)}=G x^{(n)}+H x^{(n-1)}+K
$$

Where $G=\left(1+a_{1}-b_{1}\right) I+b_{1} G_{1}$

$$
\begin{aligned}
& H=-a_{1} I \\
& K=b_{1} C
\end{aligned}
$$

$G_{m}=\left(D_{m}-L_{m}\right)^{-1} U_{m}$ be the iteration matrix of the GGS methods.

$$
C=\left(D_{m}-L_{m}\right)^{-1} b \text { is a column vector. }
$$

Using the idea of (Golub and Varga, 1961), (9) can be written in the form.

$$
\left(\begin{array}{c}
x^{(n)} \\
x^{(n+1)}
\end{array}\right)=\left(\begin{array}{cc}
0 & I \\
H & G
\end{array}\right)\left(\begin{array}{c}
x^{(n-1)} \\
x^{(n)}
\end{array}\right)+\left(\begin{array}{c}
0 \\
K
\end{array}\right)
$$

The necessary and sufficient condition for convergence of the method is that spectral radius of $\hat{G}$ must be less than unity in magnitude for any $x^{(0)}$ and $x^{(1)}$.

Using (10) and (11), for $\hat{G}=\left(\begin{array}{cc}0 & I \\ H & G\end{array}\right), \sigma(\hat{G})<1$, if and only if all roots $\lambda$ of

$$
\operatorname{det}\left(\lambda^{2} I-\lambda G-H\right)=0
$$

are less than unity in modulus.

Substituting G and H of (10) and (11) in (13), we have

$$
\operatorname{det}\left(\lambda^{2} I-\lambda\left[\left(1+a_{1}-b_{1}\right) I+b_{1} G_{m}\right]+a_{1} I\right)=0
$$

After collecting and rearranging, we have

$$
\begin{aligned}
& \operatorname{det}\left(\lambda^{2} I-\lambda\left[\left(1+a_{1}-b_{1}\right) I+b_{1} G_{m}\right]+a_{1} I\right)=0 \\
& \operatorname{det}\left(-b_{1} \lambda\left[G_{m}+\frac{\left(1+a_{1}-b_{1}\right)}{b_{1}} I-\frac{\left(\lambda^{2}+a_{1}\right)}{b_{1} \lambda} I\right)=0\right. \\
& \operatorname{det}\left(-b_{1} \lambda\left[G_{m}+\frac{\left(1+a_{1}-b_{1}\right)}{b_{1}} I-\frac{\left(\lambda^{2}+a_{1}\right)}{b_{1} \lambda} I\right)=0, \text { since } \operatorname{det}\left(-b_{1} \lambda\right)=0\right.
\end{aligned}
$$

Thus, the eigenvalues $\lambda$ of $G_{m}$ are related to the eigenvalues $\mu$ of $G_{m}$ by

$$
\mu+\frac{\left(1+a_{1}-b_{1}\right)}{b_{1}}=\frac{\left(\lambda^{2}+a_{1}\right)}{b_{1} \lambda}
$$

Let $\lambda=v e^{i \theta}$

Substituting (16) in (15), we have

$$
\mu+\frac{\left(1+a_{1}-b_{1}\right)}{b_{1}}=\frac{\left(v e^{i \theta}\right)^{2}+a_{1}}{b_{1} v e^{i \theta}}, \text { then } \mu+\frac{\left(1+a_{1}-b_{1}\right)}{b_{1}}=\frac{(v \cos \theta+i v \sin \theta)^{2}+a_{1}}{b_{1} v(\cos \theta+i \sin \theta)}
$$


After collecting and simplifying, we get

$$
\mu=\frac{\left(b_{1}-1-a_{1}\right)}{b_{1}}+\left(\frac{v^{2}+a_{1}}{b_{1} v}\right) \cos \theta+i\left(\frac{v^{2}-a_{1}}{b_{1} v}\right) \sin \theta
$$

From (17) real part of $\mu$ is $\operatorname{Re} \mu=\frac{\left(b_{1}-1-a_{1}\right)}{b_{1}}+\left(\frac{v^{2}+a_{1}}{b_{1} v}\right) \cos \theta$

Now add both sides the term $\frac{\left(1+a_{1}-b_{1}\right)}{b_{1}}$, we get

$$
\operatorname{Re} \mu+\frac{\left(1+a_{1}-b_{1}\right)}{b_{1}}=\frac{\left(b_{1}-1-a_{1}\right)}{b_{1}}+\frac{\left(1+a_{1}-b_{1}\right)}{b_{1}}+\left(\frac{v^{2}+a_{1}}{b_{1} v}\right) \cos \theta \text {. }
$$

So the result is $\operatorname{Re} \mu+\frac{\left(1+a_{1}-b_{1}\right)}{b_{1}}=\left(\frac{v^{2}+a_{1}}{b_{1} v}\right) \cos \theta$.

From this we get, $\quad \cos \theta=\frac{\operatorname{Re} \mu+\frac{\left(1+a_{1}-b_{1}\right)}{b_{1}}}{\left(\frac{v^{2}+a_{1}}{b_{1} v}\right)}$

Squaring both sides we have $\cos ^{2} \theta=\left(\frac{\operatorname{Re} \mu+\frac{\left(1+a_{1}-b_{1}\right)}{b_{1}}}{\left(\frac{v^{2}+a_{1}}{b_{1} v}\right)}\right)^{2}$.

From (17) imaginary part of $\mu$ is $\operatorname{Im} \mu=\left(\frac{v^{2}-a_{1}}{b_{1} v}\right) \sin \theta$, then we get

$$
\sin ^{2} \theta=\frac{\operatorname{Im}^{2} \mu}{\left(\frac{v^{2}-a_{1}}{b_{1} v}\right)^{2}}
$$


Add (18) and (19), we get $\frac{\left(\operatorname{Re} \mu+\frac{\left(1+a_{1}-b_{1}\right)}{b_{1}}\right)^{2}}{\left(\frac{v^{2}+a_{1}}{b_{1} v}\right)^{2}}+\frac{\operatorname{Im}^{2} \mu}{\left(\frac{v^{2}-a_{1}}{b_{1} v}\right)^{2}}=1$

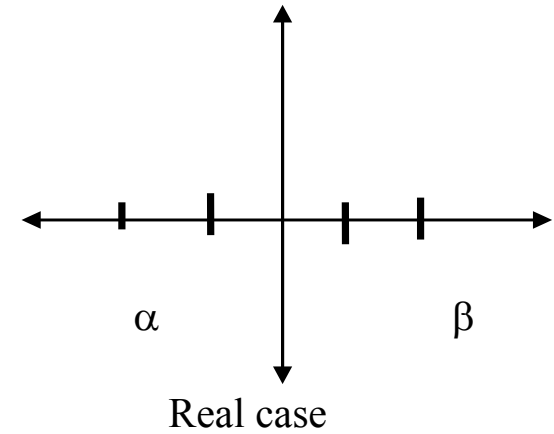

Figure1. General regions for eigenvalues $\mu$ of $G_{m}$

From (20), we have the following

$$
\frac{1}{b_{1}}\left(v+\frac{a_{1}}{v}\right)=a, \frac{1}{b_{1}}\left(v-\frac{a_{1}}{v}\right)=b,\left(\frac{b_{1}-1-a_{1}}{b_{1}}\right)=\frac{(\alpha+\beta}{2}
$$

If the eigenvalues $\mu$ of $G_{m}$ are real and lie in the interval $\alpha \leq \mu \leq \beta<1$, then the choice of $\mathrm{a}_{1}$ and $\mathrm{b}_{1}$ must satisfy the following conditions

Im $\quad 0$ (since $\mu$ is a real number), we have $\frac{1}{b_{1}}\left(v-\frac{a_{1}}{v}\right) \sin \theta=0$,

we get $\quad \mathrm{v}^{2}=\mathrm{a}$

and from figure (1) above the distance from $\beta$ to $\alpha$ is $\beta-\alpha=2 c^{\prime}$, where $c^{\prime}=\sqrt{a^{2}-b^{2}}$

Squaring both sides we get $\frac{1}{4}(\beta-\alpha)^{2}=a^{2}-b^{2}$.

Now substituting $\frac{1}{b_{1}}\left(v+\frac{a_{1}}{v}\right)$ and $\frac{1}{b_{1}}\left(v-\frac{a_{1}}{v}\right)$ in (22) for a and $\mathrm{b}$ respectively, we have $\frac{1}{4}(\beta-\alpha)^{2}=\left[\frac{1}{b_{1}}\left(v+\frac{a_{1}}{v}\right)\right]^{2}-\left[\frac{1}{b_{1}}\left(v-\frac{a_{1}}{v}\right)\right]^{2}$, then we get $\frac{1}{4}(\beta-\alpha)^{2}=\frac{4 v^{2}}{b_{1}}$, taking square root of both sides, 
we get $\quad \frac{\beta-\alpha}{2}=\frac{2 v}{b_{1}}$

Thus we have from (15), (16) and (17), we get

$$
\frac{\beta-\alpha}{2-(\beta+\alpha)}\left(1+v^{2}\right)=2 v
$$

If we let $\bar{\sigma}=\frac{\beta-\alpha}{2-(\beta+\alpha)}$, then we get

$$
\bar{\sigma}\left(1+v^{2}\right)=2 v \text { and let } 1+v^{2}=\frac{2}{1+\sqrt{1-\bar{\sigma}^{2}}}=\hat{\omega}_{b}
$$

From (16), we have

$$
\begin{gathered}
a_{1}=\hat{\omega}-1=\frac{2}{1+\sqrt{1-\bar{\sigma}^{2}}}-1=\frac{\bar{\sigma}^{2}}{\left[1+\sqrt{1-\bar{\sigma}^{2}}\right]^{2}} \\
\text { and } b_{1}=\frac{2 \bar{\sigma} \hat{\omega}_{b}}{(\beta-\alpha)}=\frac{2 \hat{\omega}_{b}}{2-(\beta+\alpha)}=\frac{4}{\left[1+\sqrt{1-\bar{\sigma}^{2}}\right][2-(\beta+\alpha)]}
\end{gathered}
$$

Therefore the spectral radius of $\hat{G}$ is $a_{1}^{\frac{1}{2}}$ (i.e. $\sigma(\hat{G})=a_{1}^{\frac{1}{2}}=\sqrt{\hat{\omega}_{b}-1}$ )

Thus with this choice of $a_{1}$ and $b_{1}$, the second degree method for any iteration is given by (David, 2007)

$$
x^{(n+1)}=\hat{\omega}_{b}\left(\frac{2 G_{m}}{2-(\beta+\alpha)}-\frac{\beta+\alpha}{2-(\beta+\alpha)} I\right) x^{(n)}+\left(1-\hat{\omega}_{b}\right) x^{(n-1)}+\frac{2 \hat{\omega}_{b}}{2-(\beta+\alpha)} C
$$

Where $\hat{\omega}_{b}=\frac{2}{1+\sqrt{1-\bar{\sigma}^{2}}}$,

$G_{m}$ is the generalized iteration matrix and $\mathrm{C}$ is column vector.

If $\mathrm{A}$ is positive definite matrix and if $G_{m} G_{m}$ is a generalized Gauss-Seidel iterative matrix and hence $\beta=\bar{\mu}^{2}, \alpha=0, \bar{\sigma}=\frac{\beta-\alpha}{2-(\beta+\alpha)}=\frac{\bar{\mu}^{2}}{2-\bar{\mu}^{2}}$, where $\bar{\mu}$ is spectral radius of generalized Jacobi matrix

$$
\hat{\omega}_{b}=\frac{2}{1+\sqrt{1-\bar{\sigma}^{2}}}=\frac{2}{1+\sqrt{1-\left(\frac{\bar{\mu}^{2}}{2-\bar{\mu}^{2}}\right)^{2}}}=\frac{4-2 \bar{\mu}^{2}}{2-\bar{\mu}^{2}+\sqrt{1-\bar{\mu}^{2}}}=\frac{2\left(2-\bar{\mu}^{2}\right)}{\left(1+\sqrt{1-\bar{\mu}^{2}}\right)^{2}}
$$




$$
\begin{aligned}
& a_{1}=\hat{\omega}_{b}-1=\frac{2}{1+\sqrt{1-\left(\frac{\bar{\mu}^{2}}{2-\bar{\mu}^{2}}\right)^{2}}}-1=\frac{\bar{\mu}^{4}}{\left(1+\sqrt{1-\bar{\mu}^{2}}\right)^{4}} \\
& \text { and } b_{1}=\frac{2 \bar{\sigma} \hat{\omega}_{b}}{(\beta-\alpha)}=\frac{2 \hat{\omega}_{b}}{2-(\beta+\alpha)}=\frac{2}{2-\bar{\mu}^{2}}\left(\frac{2\left(2-\bar{\mu}^{2}\right)}{\left(1+\sqrt{1-\bar{\mu}^{2}}\right)^{2}}\right)=\frac{4}{\left(1+\sqrt{1-\bar{\mu}^{2}}\right)^{2}} \\
& \sigma(\hat{G})=a_{1}^{\frac{1}{2}}=\sqrt{\hat{\omega}_{b}-1}=\frac{\bar{\mu}^{2}}{\left(1+\sqrt{1-\bar{\mu}^{2}}\right)^{2}}
\end{aligned}
$$

Therefore, the second degree generalized Gauss-Seidel method is given by

$$
\begin{aligned}
x^{(1)} & =G_{1} x^{(0)}+C \\
x^{(n+1)} & =\frac{2 \hat{\omega}_{b}}{2-\bar{\mu}^{2}}\left(G_{m} x^{(n)}+C_{m}\right)+\left(1-\frac{2 \hat{\omega}_{b}}{2-\bar{\mu}^{2}}\right) x^{(n)}+\left(\hat{\omega}_{b}-1\right)\left(x^{(n)}-x^{(n-1)}\right)
\end{aligned}
$$

Where $\hat{\omega}_{b}=\frac{4-2 \bar{\mu}^{2}}{2-\bar{\mu}^{2}+\sqrt{1-\bar{\mu}^{2}}}, G_{m}=\left(D_{m}-L_{m}\right)^{-1} U_{m}$ and $C_{m}=\left(D_{m}-L_{m}\right)^{-1} b_{m}$

\section{Relationship between Spectral Radiuses}

Based on the results on the spectral radius, the following relations are observed

First degree Jacobi method (FDJ) is $\bar{\mu}$.

Second degree Jacobi method (SDJ) is $\sqrt{a_{1}}=\frac{\bar{\mu}}{1+\sqrt{1-\bar{\mu}^{2}}}$.

Second degree generalized Jacobi method (SDGJ) is $\sqrt{a_{1}}=\frac{\bar{\mu}_{m}}{1+\sqrt{1-\bar{\mu}_{m}^{2}}}$

Second degree Gauss-Seidel method (SDGS) is $\sqrt{a_{1}}=\frac{\bar{\mu}^{2}}{\left(1+\sqrt{1-\bar{\mu}^{2}}\right)^{2}}$

Second degree generalized Gauss-Seidel method (SDGGS) is $\sqrt{a_{1}}=\frac{\bar{\mu}_{m}{ }^{2}}{\left(1+\sqrt{1-\bar{\mu}_{m}^{2}}\right)^{2}}$ We know $\frac{\bar{\mu}_{m}^{2}}{\left(1+\sqrt{1-\bar{\mu}_{m}^{2}}\right)^{2}} \leq \frac{\bar{\mu}_{m}}{1+\sqrt{1-\bar{\mu}_{m}^{2}}} \leq \frac{\bar{\mu}}{1+\sqrt{1-\bar{\mu}^{2}}} \leq \bar{\mu}$ Since $1+\sqrt{1-\bar{\mu}^{2}}>0$ and also $\bar{\mu}_{m}^{2} \leq \bar{\mu}_{m} \leq \bar{\mu}, \forall m=0,1,2,3 \ldots . n$, 
If $\mathrm{m}=0$, we have $\bar{\mu}_{0}=\bar{\mu}$.

\section{Numerical Examples}

Example 1: Solve the following strictly diagonal dominant (SDD) linear system of equations:

$$
\left\{\begin{array}{c}
6 x_{1}-2 x_{2}+x_{3}=1 \\
-2 x_{1}+7 x_{2}+2 x_{3}=5 \\
x_{1}+2 x_{2}-5 x_{3}=-1
\end{array}\right.
$$
Using a) GS
b) GGS
c) SDGGS

Solution:- Let us choose $\mathrm{x}^{(0)}=(0,0,0)^{\mathrm{t}}$ is an initial approximation value and tolerance number is $10^{-5}$. Now the spectral radius of Gauss-Seidel and generalized Gauss-Seidel iteration methods are $\rho(G S)=0.1396$ and $\rho($ GGS $)=0.050459$. i.e $\rho($ GGS $)<\rho(G S)$.

Example 2: Solve the following positive definite (PD) linear system of equation using GS,

GGS, SDGGS methods.

$$
\left\{\begin{array}{c}
4 x_{1}-x_{2}-x_{3}=5 \\
-x_{1}+4 x_{2}-x_{4}=-3 \\
-x_{1}+4 x_{3}-x_{4}=-7 \\
-x_{2}-x_{3}+4 x_{4}=9
\end{array}\right.
$$

Solution: Let us take the initial approximation $\mathrm{x}^{(0)}=(0,0,0,0)^{\mathrm{t}}$ with an accuracy of $10^{-5}$.

The spectral radius of Gauss-Seidel (GS) and generalized Gauss-Seidel iteration methods (GGS) are $\rho$ $(\mathrm{GS})=0.3334, \rho(\mathrm{GGS})=0.1112$. i.e $\rho(\mathrm{GGS})<\rho(\mathrm{GS})$.

\section{RESULTS AND DISCUSSION}

As presented in Table 1, the exact solution for the given linear system of equation is $(2,1,1)$. It is observed from the table that the same solution is obtained at the $8^{\text {th }}$ iteration by Gauss-Seidel method (GS), at the $6^{\text {th }}$ iteration by Generalized Gauss-Seidel method(GGS) and by Second degree Generalized Gauss-Seidel (SDGGS) method by considering only $\mathrm{m}=1$. If $\mathrm{m}=2$ one can see that at the first iteration exact solution is obtained. 
Table 1: Solution of GS, GGS and SDGGS for SDD Matrix

\begin{tabular}{cccccccccc}
\hline $\mathrm{n}$ & \multicolumn{3}{c}{$\mathrm{GS}$} & \multicolumn{3}{c}{ GGS(m=1) } & \multicolumn{3}{c}{ SDGGS(m=1) } \\
\cline { 2 - 9 } & $\mathrm{x}_{1}$ & $\mathrm{x}_{2}$ & $\mathrm{x}_{3}$ & $\mathrm{x}_{1}$ & $\mathrm{x}_{2}$ & $\mathrm{x}_{3}$ & $\mathrm{x}_{1}$ & $\mathrm{x}_{2}$ & $\mathrm{x}_{3}$ \\
\hline 0 & 0.00000 & 0.00000 & 0.00000 & 0.00000 & 0.00000 & 0.00000 & 0.00000 & 0.00000 & 0.00000 \\
1 & 1.83333 & 1.23806 & 1.06191 & 2.17890 & 1.03670 & 1.05046 & 2.17890 & 1.03670 & 1.05046 \\
2 & 2.06905 & 1.00204 & 1.01463 & 1.99097 & 0.99815 & 0.99745 & 1.98805 & 0.99759 & 0.99666 \\
3 & 1.99824 & 0.99531 & 0.99988 & 2.00044 & 1.00009 & 1.00013 & 2.00082 & 1.00016 & 1.00022 \\
4 & 1.99881 & 1.00030 & 0.99988 & 1.99998 & 1.00000 & 0.99999 & 1.99995 & 0.99999 & 0.99999 \\
5 & 2.00012 & 1.00007 & 1.00005 & 2.00000 & 1.00000 & 1.00000 & 2.00000 & 1.00000 & 1.00000 \\
6 & 2.00001 & 1.00000 & 1.00000 & 2.00000 & 1.00000 & 1.00000 & 2.00000 & 1.00000 & 1.00000 \\
7 & 2.00000 & 1.00000 & 1.00000 & & & & & & \\
8 & 2.00000 & 1.00000 & 1.00000 & & & & & & \\
\hline
\end{tabular}

As presented in Table 2, the exact solution for the given linear system of equation is $(1,0,-1,2)$. It is observed from the table that the same solution is obtained, using Gauss-Seidel method (GS) at the $12^{\text {th }}$ iteration, using Generalized Gauss-Seidel method(GGS) at the $9^{\text {th }}$ iteration and using Second degree Generalized GaussSeidel (SDGGS) method at the $6^{\text {th }}$ iteration by considering only $\mathrm{m}=1$. If $\mathrm{m}=2,3,4$ one can get almost equal to the exact solution with small number of iteration. Hence for Positive definite (PD) matrix SDGGS method is faster than first degree GS, GGS and Second degree GS method.

Table 2: Solution GS, GGS and SDGGS Iterative Methods

\begin{tabular}{|c|c|c|c|c|c|c|c|c|c|c|c|c|}
\hline \multirow[b]{2}{*}{$\mathrm{n}$} & \multicolumn{4}{|c|}{ GS } & \multicolumn{4}{|c|}{ GGS $(\mathrm{m}=1)$} & \multicolumn{4}{|c|}{ SDGGS $(m=1)$} \\
\hline & $\mathrm{x}_{1}$ & $\mathrm{x}_{2}$ & $\mathrm{x}_{3}$ & $\mathrm{x}_{4}$ & $\mathrm{x}_{1}$ & $\mathrm{x}_{2}$ & $\mathrm{x}_{3}$ & $\mathrm{x}_{4}$ & $\mathrm{x}_{1}$ & $\mathrm{x}_{2}$ & $\mathrm{x}_{3}$ & $\mathrm{x}_{4}$ \\
\hline 0 & 0.00000 & 0.00000 & 0.00000 & 0.00000 & 0.00000 & 0.00000 & 0.00000 & 0.00000 & 0.00000 & 0.00000 & 0.00000 & 0.00000 \\
\hline 1 & 1.25000 & 0.43750 & -1.4375 & 1.78125 & 1.13333 & 0.46667 & -0.99556 & 1.88444 & 1.13333 & 0.46667 & -0.9955 & 1.88444 \\
\hline 2 & 0.78125 & 0.10938 & -1.9453 & 1.94531 & 0.99348 & 0.03052 & -1.00377 & 1.99143 & 0.98610 & 0.00485 & -1.0051 & 1.99946 \\
\hline 3 & . & . & . & . & . & . & . & . & 0.99921 & 0.00018 & -1.0001 & 1.99990 \\
\hline 4 & . & . & . & . & . & . & . & . & 1.00001 & 0.00005 & -1.0000 & 1.99999 \\
\hline 5 & . & . & . & . & . & . & . & . & 1.00000 & 0.00001 & -1.0000 & 2.00000 \\
\hline 6 & . & . & . & . & . & . & . & . & 1.00000 & 0.00000 & -1.0000 & 2.00000 \\
\hline 7 & . & . & . & . & . & . & . & . & & & & \\
\hline 8 & . & . & . & . & 1.00000 & 0.000000 & -1.00000 & 1.999999. & & & & \\
\hline 10 & . & . & . & . & & & & & & & & \\
\hline 11 & 1.00000 & 0.00000 & -1.0000 & 2.00000 & & & & & & & & \\
\hline 12 & 1.00000 & 0.00000 & -1.0000 & 2.00000 & & & & & & & & \\
\hline
\end{tabular}




\section{CONCLUSION}

If matrix A is strictly diagonal dominate (SDD) and positive definite (PD) matrix, then by the use of second degree generalized Gauss-Seidel iterative method(SDGGS), it is possible to accelerate the convergence rate of the solution of linear system of equations which has real and complex eigenvalues that are less than unity in magnitude. The numerical results shows that the SDGGS method is more effective than first degree GaussSeidel (GS), first degree generalized Gauss-Seidel (GGS) and second degree Gauss-Seidel (GS) methods. Moreover, from the relationship of spectral radius, the spectral radius of SDGGS is less than SDGS. In general, the results of numerical examples and spectral radius comparison considered clearly demonstrate the accuracy of the methods developed in this article. It is conjectured that the rate of convergence of some methods developed in this paper can be further enhanced by using extrapolating techniques.

\section{ACKNOWLEDGEMENTS}

I would like to express my sincere appreciation to Professor Vatti, Bassava Kumar, Department of Engineering mathematics, College of Engineering, Andhra University and Gashaye Dessalew for their comments and suggestion on the article.

\section{REFERENCES}

David, M. Y. (1970). Second-degree iterative methods for the solution of large linear systems. Journal of Approximation Theory 5:137-148.

David, K. S. (2007). Generalized Jacobi and Gauss-Seidel methods for solving linear system of equations. Numerical Mathematics, A Journal of Chinese Universities. (English Series) 16: 164-170.

Golub, G.H and Varga, R.S. (1961). Chebyshev semi-iterative methods, Successive Over-Relaxation iterative methods and second-order Richardson iterative Methods. Numerical Mathematics.

Kahan, W. (1958). Gauss-Seidel methods for solving large systems of linear equations. PhD Thesis, University of Toronto.

Li, W. (2005). A note on the preconditioned (GS) method for Large system of equation. Journal of Computational Applied Mathematics 182:81-90.

Saad, Y. (1995). Iterative methods for sparse linear systems. PWS Press, New York. 